Crystal and Molecular Structure Determination $\left[\mathrm{Ni}_{5} \mathrm{Sb}_{17}\right]\left[\mathrm{K}\left(\mathrm{C}_{18} \mathrm{H}_{36} \mathrm{~N}_{2} \mathrm{O}_{6}\right)\right]_{4}\left[\mathrm{C}_{2} \mathrm{H}_{8} \mathrm{~N}_{2}\right]$

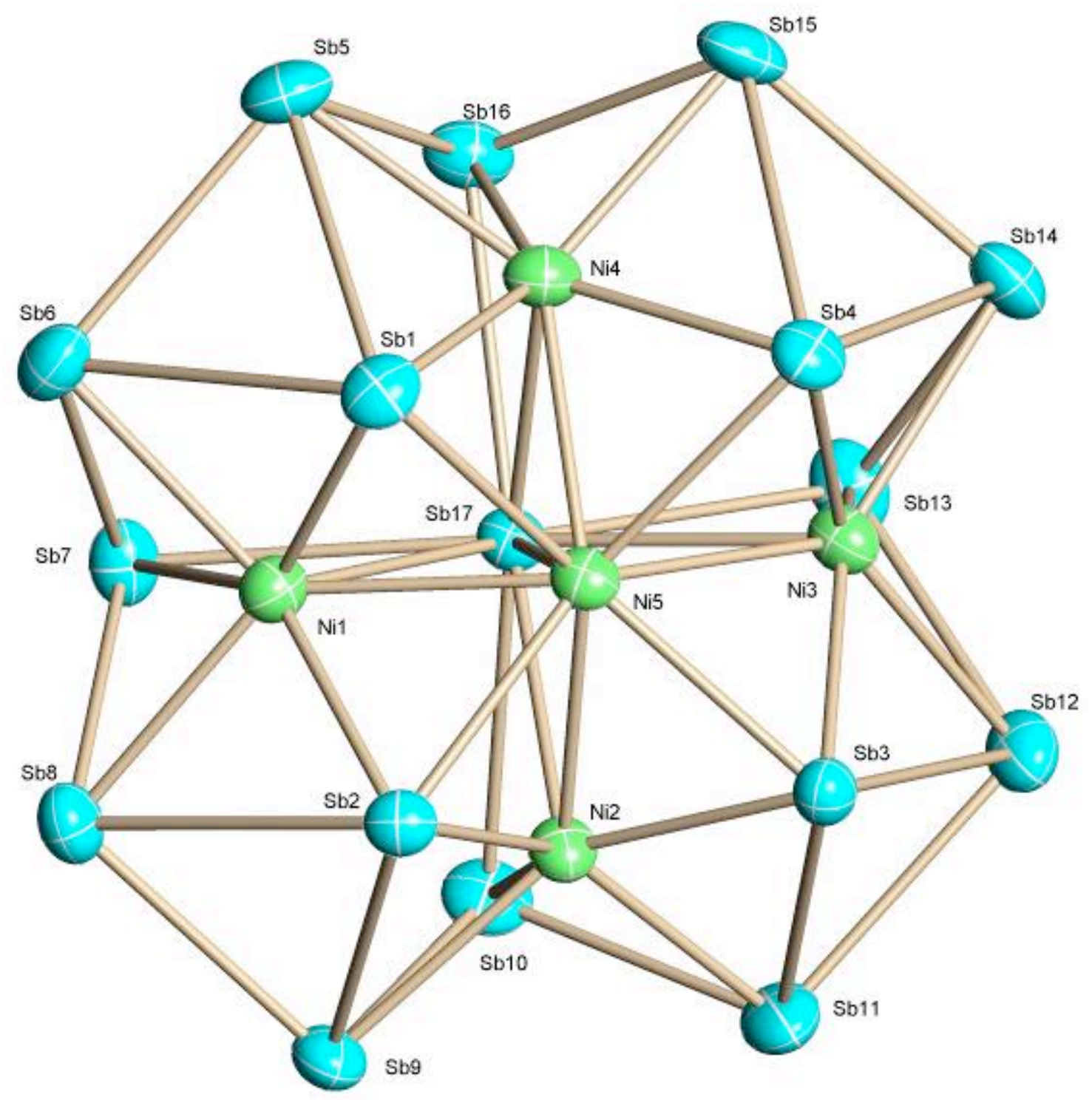


A black block with approximate orthogonal dimensions $0.204 \times 0.146 \times 0.040 \mathrm{~mm}^{3}$ was placed and optically centered on the Bruker SMART CCD system at $-100^{\circ} \mathrm{C}$. The initial unit cell was indexed using a least-squares analysis of a random set of reflections collected from three series of $0.3^{\circ}$ wide $\omega$-scans, 10 seconds per frame, and 25 frames per series that were well distributed in reciprocal space. Data frames were collected $[\mathrm{MoK} \alpha]$ with $0.3^{\circ}$ wide $\omega$-scans, 40 seconds per frame and 606 frames per series. Six data series were collected, five at varying $\varphi$ angles $\left(\varphi=0^{\circ}, 72^{\circ}\right.$, $144^{\circ}, 216^{\circ}, 288^{\circ}$ ), a sixth composed of $9090.3^{\circ}$ wide $\omega$ scans with fixed $\varphi=0^{\circ}$ and finally, 200 frames, a partial repeat of the first series for decay purposes. The crystal to detector distance was $4.371 \mathrm{~cm}$, thus providing a complete sphere of data to $2 \theta_{\max }=50.0^{\circ}$. A total of 42595 reflections were collected and corrected for Lorentz and polarization effects and absorption using Blessing's method as incorporated into the program SADABS $^{1,2}$ with 21436 unique $[\mathrm{R}(\mathrm{int})=0.0334]$

\section{Structural determination and Refinement:}

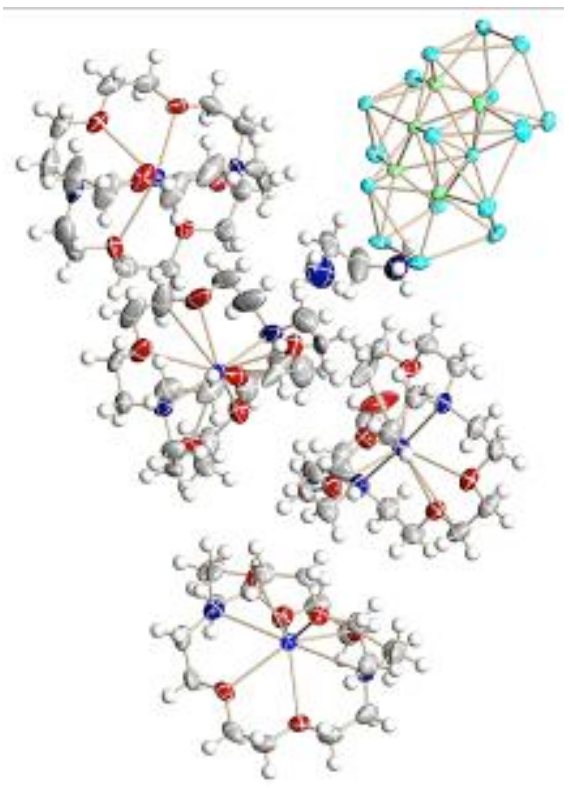

All crystallographic calculations were performed on a Personal computer (PC) with a Pentium $1.80 \mathrm{GHz}$ processor and 512MB of extended memory. The SHELXTL ${ }^{3}$ program package was implemented to determine the probable space group and set up the initial files. System symmetry, lack of systematic absences and intensity statistics indicated the centrosymmetric triclinic space group P-1 (no. 2). The structure was determined by direct methods with the successful location of nearly the entire molecule of interest and the central potassium atoms of the cations using the program $\mathrm{XS}^{4}$. The structure was refined with $\mathrm{XL}^{5}$. A series of least-squares difference-Fourier cycles were required to locate the remaining non-hydrogen atoms. All non-hydrogen atoms were refined anisotropically. An ethylenediamine molecule was also located. The $\left[\mathrm{Ni}_{5} \mathrm{Sb}_{17}\right]^{4-}$ molecule was found to possess a rotational disorder wherein two complete molecules were refined occupying the same location with the final ratio $0.8125: 0.1875$. All of the hydrogen atoms were placed in calculated positions throughout the refinement. The final structure was refined to convergence $[\Delta / \sigma \leq 0.001]$ with $\mathrm{R}(\mathrm{F})=7.68 \%, \mathrm{wR}\left(\mathrm{F}^{2}\right)=11.68 \%, \mathrm{GOF}=1.074$ for all 21436 unique reflections $\left[\mathrm{R}(\mathrm{F})=4.43 \%, \mathrm{wR}\left(\mathrm{F}^{2}\right)=10.72 \%\right.$ for those 15133 data with $\mathrm{Fo}>$ $4 \sigma(\mathrm{Fo})]$. The final difference-Fourier map was featureless indicating that the structure is both correct and complete.

The function minimized during the full-matrix least-squares refinement was $\Sigma \mathrm{w}\left(\mathrm{Fo}^{2}-\mathrm{Fc}^{2}\right)$ where $\mathrm{w}=1 /\left[\mathrm{\sigma}^{2}\left(\mathrm{Fo}^{2}\right)+(0.0491 * \mathrm{P})^{2}+022.2174 * \mathrm{P}\right]$ and $\mathrm{P}=\left(\max \left(\mathrm{Fo}^{2}, 0\right)+2 * \mathrm{Fc}^{2}\right) / 3$. An empirical correction for extinction was also applied to the data in the form $\left(\mathrm{Fc}^{2}, \mathrm{corr}\right)=$ 
$\mathrm{k}\left[1+0.001 * \mathrm{x} * \mathrm{Fc}^{2} * \lambda^{3} / \sin (2 \theta)\right]^{(-1 / 4)}$ where $\mathrm{k}=0.09959$ is the overall scale factor. The value determined for $\mathrm{x}$ was $0.000014(13)$.

\section{References:}

1. An Empirical Correction for Absorption Anisotropy, Blessing, R. H. (1995). Acta Cryst., A51, 33-38.

2. Sheldrick, G.M., SADABS 'Siemens Area Detector Absorption Correction' Universität Göttingen: Göttingen, Germany, 1996.

3. Sheldrick, G.M., (1994). SHELXTL/PC. Version 5.03. Siemens Analytical X-ray Instruments Inc., Madison, Wisconsin, USA.

4. Phase Annealing in SHELX-90: Direct Methods for Larger Structures, Sheldrick, G. M., (1990). Acta Cryst. A46, 467-473.

5. Sheldrick, G.M., (1993). Shelx193 Program for the Refinement of Crystal Structures. University of Göttingen, Germany. 
Table S1. Crystal data and structure refinement for $\left[\mathrm{Ni}_{5} \mathrm{Sb}_{17}\right]\left[\mathrm{K}\left(\mathrm{C}_{18} \mathrm{H}_{36} \mathrm{~N}_{2} \mathrm{O}_{6}\right)\right]_{4}\left[\mathrm{C}_{2} \mathrm{H}_{8} \mathrm{~N}_{2}\right]$.

Identification code

Empirical formula

Formula weight

Temperature

Wavelength

Crystal system

Space group

Unit cell dimensions

Volume

$\mathrm{Z}$

Density (calculated)

Absorption coefficient

$\mathrm{F}(000)$

Crystal size

Theta range for data collection

Index ranges

Reflections collected

Independent reflections

Completeness to theta $=25.00^{\circ}$

Absorption correction

Max. and min. transmission

Refinement method

Data / restraints / parameters

Goodness-of-fit on $\mathrm{F}^{2}$

Final $\mathrm{R}$ indices [I $>2 \operatorname{sigma}(\mathrm{I})]$

$\mathrm{R}$ indices (all data)

Extinction coefficient

Largest diff. peak and hole 991ffmi

C74 H152 K4 N10 Ni5 O24 Sb17

4085.76

173(2) K

$0.71073 \AA$

Triclinic

P-1

$$
\begin{array}{ll}
\mathrm{a}=15.510(3) \AA & \alpha=86.253(4)^{\circ} . \\
\mathrm{b}=16.661(3) \AA & \beta=81.860(4)^{\circ} . \\
\mathrm{c}=25.943(5) \AA & \gamma=66.513(3)^{\circ} .
\end{array}
$$

6086.3(19) $\AA^{3}$

2

$2.229 \mathrm{Mg} / \mathrm{m}^{3}$

$4.65 \mathrm{~mm}^{-1}$

3882

$0.20 \times 0.15 \times 0.04 \mathrm{~mm}^{3}$

1.59 to $25.00^{\circ}$.

$-18<=\mathrm{h}<=18,-19<=\mathrm{k}<=19,-30<=\mathrm{l}<=30$

42595

$21436[\mathrm{R}(\mathrm{int})=0.0334]$

$100.0 \%$

Empirical, SADABS (multi-scan)

0.8358 and 0.4505

Full-matrix least-squares on $\mathrm{F}^{2}$

21436 / 0 / 1390

1.074

$\mathrm{R} 1=0.044, \mathrm{wR} 2=0.107$ [15133 Data]

$\mathrm{R} 1=0.077, \mathrm{wR} 2=0.117$

$0.000014(13)$

1.15 and -1.15 e. $\AA^{-3}$ 
$\underline{\text { Table S-2}}$. Select bond distances $(\AA)$ and angles $\left(^{\circ}\right)$ for $\left[\mathrm{Ni}_{5} \mathrm{Sb}_{17}\right]^{-4}$.

\begin{tabular}{|c|c|c|c|}
\hline Sb1-Sb5 & $3.135(3)$ & Ni4-Sb5 & $2.540(2)$ \\
\hline Sb1-Sb6 & $3.152(2)$ & Ni4-Sb15 & $2.525(3)$ \\
\hline $\mathrm{Sb} 2-\mathrm{Sb} 8$ & $3.150(5)$ & $\mathrm{Ni4}-\mathrm{Sb} 16$ & $2.549(2)$ \\
\hline $\mathrm{Sb} 2-\mathrm{Sb} 9$ & $3.095(2)$ & Ni4-Sb17 & $2.8206(15)$ \\
\hline Sb3-Sb11 & $3.097(4)$ & Ni5-Sb1 & $2.590(2)$ \\
\hline $\mathrm{Sb} 3-\mathrm{Sb} 12$ & $3.152(3)$ & Ni5-Sb2 & $2.613(3)$ \\
\hline Sb4-Sb14 & $3.125(2)$ & Ni5-Sb3 & $2.592(2)$ \\
\hline Sb4-Sb15 & $3.107(4)$ & Ni5-Sb4 & $2.604(3)$ \\
\hline Sb5-Sb6 & $2.881(3)$ & Ni5-Sb17 & $2.5430(15)$ \\
\hline $\mathrm{Sb} 5-\mathrm{Sb} 16$ & $2.823(3)$ & Ni5-Ni1 & $2.552(2)$ \\
\hline Sb6-Sb7 & $2.833(2)$ & Ni5-Ni2 & $2.551(2)$ \\
\hline $\mathrm{Sb} 7-\mathrm{Sb} 8$ & $2.816(4)$ & Ni5-Ni3 & $2.590(2)$ \\
\hline Sb7-Sb17 & $3.1396(16)$ & Ni5-Ni4 & $2.540(2)$ \\
\hline Sb8-Sb9 & $2.901(4)$ & Ni3-Sb3 & $2.5142(17)$ \\
\hline Sb9-Sb10 & $2.856(2)$ & $\mathrm{Ni3}-\mathrm{Sb} 4$ & $2.524(2)$ \\
\hline Sb10-Sb11 & $2.842(5)$ & $\mathrm{Ni3}-\mathrm{Sb} 12$ & $2.552(2)$ \\
\hline Sb10-Sb17 & $3.1667(17)$ & $\mathrm{Ni3}-\mathrm{Sb} 13$ & $2.553(3)$ \\
\hline $\mathrm{Sb} 11-\mathrm{Sb} 12$ & $2.919(4)$ & Ni3-Sb14 & $2.5472(19)$ \\
\hline $\mathrm{Sb} 12-\mathrm{Sb} 13$ & $2.851(4)$ & Ni3-Sb17 & $2.8445(16)$ \\
\hline Sb13-Sb14 & $2.847(3)$ & Ni4-Sb1 & $2.503(2)$ \\
\hline Sb13-Sb17 & $3.176(2)$ & Ni4-Sb4 & $2.510(2)$ \\
\hline Sb14-Sb15 & $2.928(4)$ & & \\
\hline
\end{tabular}




\begin{tabular}{|c|c|c|c|}
\hline $\mathrm{Sb} 15-\mathrm{Sb} 16$ & $2.828(4)$ & $\mathrm{Sb} 2-\mathrm{Ni1}-\mathrm{Sb} 8$ & $77.48(9)$ \\
\hline Sb16-Sb17 & $3.1577(18)$ & $\mathrm{Sb} 2-\mathrm{Ni1}-\mathrm{Sb} 6$ & $145.73(8)$ \\
\hline Ni1-Sb1 & $2.5057(19)$ & $\mathrm{Sb} 7-\mathrm{Ni1}-\mathrm{Sb} 8$ & $67.20(10)$ \\
\hline Ni1-Sb2 & $2.496(3)$ & Ni5-Ni1-Sb7 & $127.54(6)$ \\
\hline Ni1-Sb6 & $2.547(2)$ & Ni1-Ni5-Sb17 & $67.24(5)$ \\
\hline Ni1-Sb7 & $2.5509(19)$ & Ni1-Ni5-Ni2 & $79.53(7)$ \\
\hline Ni1-Sb8 & $2.537(5)$ & Ni1-Ni5-Ni3 & $134.55(7)$ \\
\hline Ni1-Sb17 & $2.8213(15)$ & $\mathrm{Sb} 5-\mathrm{Sb} 1-\mathrm{Sb} 6$ & $54.55(5)$ \\
\hline $\mathrm{Ni} 2-\mathrm{Sb} 2$ & $2.522(2)$ & $\mathrm{Sb6-Sb7-Sb8}$ & $83.22(10)$ \\
\hline Ni2-Sb3 & $2.5107(17)$ & $\mathrm{Sb} 7-\mathrm{Sb} 8-\mathrm{Sb} 9$ & $102.92(15)$ \\
\hline Ni2-Sb9 & $2.541(2)$ & Sb1-Ni5-Sb17 & $105.40(8)$ \\
\hline Ni2-Sb10 & $2.542(2)$ & Sb1-Ni5-Sb4 & $86.45(8)$ \\
\hline Ni2-Sb11 & $2.527(4)$ & Sb1-Ni1-Sb2 & $89.41(6)$ \\
\hline $\mathrm{Ni2}-\mathrm{Sb} 17$ & $2.7825(16)$ & Sb1-Ni1-Sb6 & $77.20(6)$ \\
\hline
\end{tabular}


Table S-3. Select bond distances $(\AA)$ and angles $\left(^{\circ}\right)$ for $\left[\mathrm{Pd}_{7} \mathrm{As}_{16}\right]^{-4}$.

\begin{tabular}{|c|c|c|c|}
\hline As1-As5 & $2.8145(14)$ & Pd1-Pd6 & $2.8213(10)$ \\
\hline As1-As6 & $2.8326(15)$ & $\mathrm{Pd} 2-\mathrm{Pd} 5$ & $2.8434(9)$ \\
\hline As2-As11 & $2.4210(15)$ & $\mathrm{Pd} 2-\mathrm{Pd} 6$ & $2.8242(10)$ \\
\hline As3-As12 & $2.7940(14)$ & $\mathrm{Pd} 3-\mathrm{Pd} 5$ & $2.8292(10)$ \\
\hline As3-As13 & $2.7521(13)$ & $\mathrm{Pd} 3-\mathrm{Pd} 7$ & $2.8516(10)$ \\
\hline As4-As10 & $2.4298(15)$ & $\mathrm{Pd} 4-\mathrm{Pd} 5$ & $2.8394(9)$ \\
\hline As5-As6 & $2.4407(14)$ & $\mathrm{Pd} 4-\mathrm{Pd} 7$ & $2.7999(9)$ \\
\hline As5-As9 & $2.4747(14)$ & Pd6-Pd7 & $2.8768(9)$ \\
\hline As6-As7 & $2.4883(15)$ & & \\
\hline As7-As8 & $2.4185(14)$ & As5-As1-As6 & $51.21(3)$ \\
\hline As8-As9 & $2.4235(13)$ & As6-As5-As9 & $106.99(5)$ \\
\hline As12-As13 & $2.4392(13)$ & As5-As6-As7 & $108.40(5)$ \\
\hline As12-As16 & $2.4815(14)$ & As6-As7-As8 & $107.05(5)$ \\
\hline As13-As14 & $2.4948(13)$ & As7-As8-As9 & $109.30(5)$ \\
\hline As14-As15 & $2.4114(13)$ & As8-As9-As5 & $108.22(5)$ \\
\hline As15-As16 & $2.4099(13)$ & As11-As2-Pd5 & $106.81(5)$ \\
\hline Pd1-As1 & $2.5013(12)$ & Pd1-As11-Pd2 & $90.86(4)$ \\
\hline Pd1-As2 & $2.4985(12)$ & As2-As11-Pd6 & $102.47(4)$ \\
\hline Pd1-As6 & $2.4433(11)$ & As2-Pd1-As1 & $90.93(4)$ \\
\hline Pd1-As7 & $2.5416(13)$ & As6-Pd1-As2 & $153.20(5)$ \\
\hline Pd1-As11 & $2.5082(11)$ & As6-Pd1-As7 & $59.85(4)$ \\
\hline Pd5-As1 & $2.5984(11)$ & As11-Pd1-As2 & $57.83(4)$ \\
\hline
\end{tabular}




$\begin{array}{llll}\text { Pd5-As2 } & 2.5107(11) & \text { As4-Pd5-As2 } & 161.70(5) \\ \text { Pd5-As3 } & 2.6343(10) & \text { As2-Pd5-As1 } & 88.45(4) \\ \text { Pd5-As4 } & 2.5485(11) & \text { As7-Pd6-As11 } & 85.03(4) \\ \text { Pd6-As7 } & 2.6043(11) & \text { Pd1-Pd5-Pd4 } & 62.57(3) \\ \text { Pd6-As8 } & 2.5857(11) & \text { Pd2-Pd5-Pd1 } & 76.89(2) \\ \text { Pd6-As11 } & 2.6176(12) & \text { Pd1-Pd6-Pd2 } & 78.28(3) \\ \text { Pd1-Pd5 } & 2.8876(10) & \text { Pd1-Pd6-Pd7 } & 97.55(3)\end{array}$




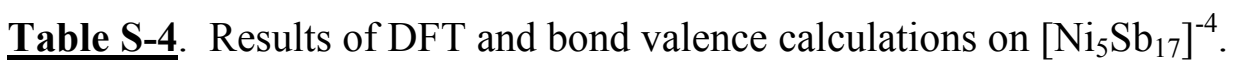

\begin{tabular}{lllllll} 
& \multicolumn{3}{c}{ Bond Order } & \multicolumn{3}{c}{ Formal Charge } \\
Atom & BVF & DFT & $\Delta$ & BVF & DFT & $\Delta$ \\
Sb1 & 1.148 & 1.54 & -0.392 & -1.852 & -0.1726 & -1.6794 \\
Sb3 & 1.181 & 1.50 & -0.319 & -1.819 & -0.1873 & -1.6317 \\
Sb4 & 1.187 & 1.49 & -0.303 & -1.813 & -0.1888 & -1.6242 \\
Sb2 & 1.201 & 1.52 & -0.319 & -1.799 & -0.1750 & -1.624 \\
Sb17 & 1.630 & 1.02 & 0.61 & -1.370 & 0.6441 & -2.0141 \\
Sb12 & 2.152 & 2.26 & -0.108 & -0.848 & -0.2569 & -0.5911 \\
Sb14 & 2.154 & 2.26 & -0.106 & -0.846 & -0.2536 & -0.5924 \\
Sb9 & 2.232 & 2.25 & -0.018 & -0.768 & -0.2502 & -0.5178 \\
Sb15 & 2.232 & 2.27 & -0.038 & -0.768 & -0.2517 & -0.5163 \\
Sb11 & 2.239 & 2.26 & -0.021 & -0.761 & -0.2547 & -0.5063 \\
Sb8 & 2.276 & 2.26 & 0.016 & -0.724 & -0.2496 & -0.4744 \\
Sb13 & 2.282 & 1.93 & 0.352 & -0.718 & -0.3725 & -0.3455 \\
Sb10 & 2.294 & 1.93 & 0.364 & -0.706 & -0.3695 & -0.3365 \\
Sb6 & 2.295 & 2.25 & 0.045 & -0.705 & -0.2524 & -0.4526 \\
Sb5 & 2.334 & 2.25 & 0.084 & -0.666 & -0.2544 & -0.4116 \\
Sb16 & 2.437 & 1.93 & 0.507 & -0.563 & -0.3703 & -0.1927 \\
Sb7 & 2.460 & 1.93 & 0.53 & -0.540 & -0.3657 & -0.1743
\end{tabular}


$\underline{\text { Table S-5. }}$. Results of DFT and bond valence calculations on $\left[\mathrm{Pd}_{7} \mathrm{As}_{16}\right]^{-4}$.

\begin{tabular}{lllllll} 
& \multicolumn{3}{c}{ Bond Order } & \multicolumn{3}{c}{ Formal Charge } \\
Atom & BVF & DFT & $\Delta$ & BVF & DFT & \multicolumn{1}{c}{$\Delta$} \\
As15 & 0.679 & 1.64 & -0.961 & -2.321 & -0.6073 & -1.7137 \\
As16 & 0.830 & 1.64 & -0.81 & -2.170 & -0.6080 & -1.562 \\
As14 & 1.000 & 1.69 & -0.69 & -2.000 & -0.7359 & -1.2641 \\
As13 & 1.025 & 1.69 & -0.665 & -1.975 & -0.7328 & -1.2422 \\
As12 & 1.029 & 1.75 & -0.721 & -1.971 & -0.6400 & -1.331 \\
As11 & 1.127 & 1.75 & -0.623 & -1.873 & -0.6361 & -1.2369 \\
As10 & 1.877 & 2.38 & -0.503 & -1.123 & -0.4980 & -0.625 \\
As3 & 1.887 & 2.39 & -0.503 & -1.113 & -0.4980 & -0.615 \\
As8 & 1.923 & 2.39 & -0.467 & -1.077 & -0.4972 & -0.5798 \\
As5 & 1.930 & 2.39 & -0.46 & -1.070 & -0.4974 & -0.5726 \\
As4 & 2.085 & 2.37 & -0.285 & -0.915 & -0.3950 & -0.52 \\
As2 & 2.105 & 2.48 & -0.375 & -0.895 & -0.4394 & -0.4556 \\
As9 & 2.139 & 2.37 & -0.231 & -0.861 & -0.3958 & -0.4652 \\
As6 & 2.165 & 2.47 & -0.305 & -0.835 & -0.4376 & -0.3974 \\
As1 & 2.170 & 2.48 & -0.31 & -0.830 & -0.4385 & -0.3915 \\
As7 & 2.185 & 2.48 & -0.295 & -0.815 & -0.4384 & -0.3766
\end{tabular}




\section{Experimental Section}

General Data. All reactions were carried out in a nitrogen atmosphere dry box (Vacuum Atmosphere Co). Electrospray Mass spectra were obtained by direct injection of dmf solutions into a Finnigan mass spectrometer. Samples were detected in the negative ion mode. An AMRAY $1820 \mathrm{~K}$ scanning electron microscope with a potential of $20 \mathrm{kV}$ was used for energy dispersive X-ray (EDX) studies.

Chemicals. Melts of nominal composition of $\mathrm{K}_{3} \mathrm{Sb}_{7}$ were prepared by fusion of stoichiometric ratios of the elements at high temperature $\left(\sim 1100^{\circ} \mathrm{C}\right)$. The elements were loaded into quartz tubes in a nitrogen atmosphere dry box and then sealed under vacuum. CAUTION: the fusion process can be very exothermic and the reactions should be conducted behind blast shields on small scales $(<1 \mathrm{~g})$ using full protective gear. $\mathrm{Ni}(\mathrm{COD})_{2}$ was purchased from STREM Chemicals (COD = cyclooctadiene). 4,7,13,16,21,24-hexaoxa-1,10-diazobicyclo[8.8.8] hexacosane $(2,2,2-$ crypt) was purchased from Aldrich. Anhydrous ethylenediamine (en) and dimethylformamide (dmf) were purchased from Fischer, vacuum distilled from $\mathrm{K}_{4} \mathrm{Sn}_{9}$ and stored under $\mathrm{N}_{2}$. Toluene was distilled from sodium under dinitrogen and stored under $\mathrm{N}_{2}$.

Preparation of $[\mathbf{K}(\mathbf{2}, \mathbf{2}, \mathbf{2}-\mathbf{c r y p t})]_{4}\left[\mathbf{N i}_{5} \mathrm{Sb}_{17}\right] \cdot \mathbf{e n ~} \mathrm{K}_{3} \mathrm{Sb}_{7}(50 \mathrm{mg}, 0.052 \mathrm{mmol}), \mathrm{Ni}(\mathrm{COD})_{2}(12 \mathrm{mg}$, $0.044 \mathrm{mmol}$ ) and 2,2,2-crypt (34 mg, $0.090 \mathrm{mmol})$ were dissolved in en $(\sim 2.5 \mathrm{~mL})$ and stirred for 7h. After stirring, the solution was filtered through tightly packed glass wool. The filtered solution was heated at $\sim 50{ }^{\circ} \mathrm{C}$ for $8 \mathrm{~min}$. and filtered through heated glassware. Solution was allowed to cool on the shelf for $24-48 \mathrm{~h}$ at which time small black crystals of [K(2,2,2crypt $)]_{4}\left[\mathrm{Ni}_{5} \mathrm{Sb}_{17}\right] \cdot e n$ co-crystallizes with $[\mathrm{K}(2,2,2 \text {-crypt })]_{4}\left[\left(\mathrm{Sb}_{7}\right)_{2}\left(\mathrm{Ni}_{2} \mathrm{Sb}_{2}\right)\right] \cdot 2 \mathrm{en}$, in low yield 